\title{
Prevalence and intensity of intestinal helminths found in free-ranging golden lion tamarins (Leontopithecus rosalia, Primates, Callitrichidae) from Brazilian Atlantic forest
}

\author{
Rafael V. Monteiro $^{\mathrm{a}, *}$, James M. Dietz ${ }^{\mathrm{b}}$, Benjamin B. Beck ${ }^{\mathrm{c}}$, Andrew J. Baker ${ }^{\mathrm{d}}$, \\ Andreia Martins $^{\mathrm{e}}$, Ana Maria Jansen ${ }^{\mathrm{a}}$ \\ ${ }^{a}$ Instituto Oswaldo Cruz - FIOCRUZ, Lab. Biologia de Tripanosomatídeos, Pavilhão Carlos Chagas $3^{\circ}$ andar, \\ Av. Brasil 4365 - Rio de Janeiro, RJ 21040-360, Brazil \\ ${ }^{\mathrm{b}}$ Department of Biology, University of Maryland, College Park, MD 20742, USA \\ ${ }^{\mathrm{c}}$ Great Ape Trust of Iowa, 4200 S.E. 44th Avenue, Des Moines, IA 50320, USA \\ ${ }^{\mathrm{d}}$ Philadelphia Zoo, 3400 West Girard Avenue, Philadelphia, PA 19104-1196, USA \\ e Associação Mico Leão Dourado, Caixa Postal 109.968, Casimiro de Abreu, RJ 28960-970, Brazil
}

Received 25 August 2006; received in revised form 29 November 2006; accepted 5 December 2006

\begin{abstract}
Helminth identification and egg counts were performed in 316 fecal samples during 4 years in 199 golden lion tamarins (GLTs), Leontopithecus rosalia, from two Brazilian conservation units. Tamarin sex and age, area of occurrence and helminth co-infection were tested as potential factors that could affect helminth prevalence and egg shedding in host individuals. Three nematodes species were found at low prevalences but not in both conservation units: Ancylostomatidae (12\%), Ascarididae (1\%) and Tripanoxyuris minutus, an Oxyuridae (4\%). Three other species had high prevalences and were found in both conservation units: one acanthocephalan, Oncicola sp. (30\%), and two nematodes, Spiruridae (24\%), and Trichostrongylidae (31\%). These three latter helminths had distinct prevalences between the conservation units, probably due to differences in availability of helminth infective stages in each area. Prevalences were greater in females for all helminth species; this was especially the case for Oncicola sp. Sex and age differences in helminth prevalence may be associated with changes in sexual steroid levels that accompany age and reproductive status. Frequency of simultaneous infections by the two helminths considered most pathogenic (Oncicola and Trichostrongylidae) were statistically lower than expected; this may be related to: (i) higher tamarin death rate caused by the association; (ii) differences in exposure to helminth infective stages in the various areas of occurrence; (iii) and competition between these helminth species. All helminth species followed a negative binomial distribution, with stronger clumping occurring in Trichostrongylidae and in female GLTs. Distinct transmission strategies of Oncicola sp. and Spiruridae, in contrast with Trichostrongylidae, may partially explain the different clumping levels of these helminths in GLTs. Mean fecal egg counts of all helminths were not different between GLT sexes, ages or areas of occurrence. The three most common helminth species may be a threat to isolated or dense GLT populations.
\end{abstract}

(C) 2006 Elsevier B.V. All rights reserved.

Keywords: Wildlife epidemiology; Free-ranging primates; Wild animals diseases; Endangered species; Conservation medicine; Parasite aggregation; Lion tamarins; Helminth

* Corresponding author. Tel.: +55 212598 4324; fax: +55 2125606572.
E-mail address: rafael.monteiro@infolink.com.br (R.V. Monteiro).

0304-4017/\$ - see front matter (C) 2006 Elsevier B.V. All rights reserved.

doi:10.1016/j.vetpar.2006.12.004 


\section{Introduction}

Helminth prevalence is thought to follow a negative binomial distribution in host populations (Anderson and May, 1985), i.e., a few individuals in the host population carry the majority of the helminth population. This clumped distribution pattern is generated by the influence of biotic and abiotic factors that modify the host-parasite relationship through time (Wilson et al., 2002). Several such factors have been identified as potential causes of this variation. Sex steroid hormones (estrogen, progesterone and testosterone) are thought to modulate host immunoresponse in humans (Bouman et al., 2005) and in animal models (Klein, 2004). Sex, age and reproductive status, as factors that alter the expression of such hormones, may indirectly modulate disease susceptibility and severity. Thus, male mammals may have lower immune response when secondary reproductive characters develop and/or when they assume a reproductive position in group-living species. Females may have a lower immune status when they begin to reproduce (Lamason et al., 2006).

Host geographic area of occurrence may also affect prevalence and incidence of helminthic diseases. Factors such as floristic composition, humidity, sun exposure and presence or absence of invertebrate vectors could affect the viability and availability of helminth eggs, resulting in variation in the prevalence of infection (Gulland, 1995). Host age is also implicated in causing differences in helminth distribution. The peak shift theory states that as the rate of transmission increases, immunity and the peak of prevalence will develop at an earlier age in the host population (Woolhouse, 1998). Finally, inter- and intraspecific interactions among helminths inside the intestinal tract of the host may influence the prevalence of each (Poulin, 2001).

In the present longitudinal study, we evaluated the influence of host sex, age, area of occurrence and helminth co-infection as sources of variation in helminth prevalence and egg-shedding intensity in free-ranging golden lion tamarins (GLT), Leontopithecus rosalia (Primates: Callitrichidae). This species is endemic to Brazil's Atlantic forest, a region considered one of the world hotspots for conservation efforts (Myers et al., 2000). GLTs were on the verge of extinction 20 years ago, but intense conservation work resulted in the recent lowering of its threat status from critically endangered to endangered (IUCN, 2004). An evaluation of the demographic effects of helminth infection on golden lion tamarin populations is an additional objective of our research.

\section{Material and methods}

\subsection{Animals and study areas}

Subjects in this study included GLTs from a biological reserve and several small, privately owned forest islands. The 6300 ha Poço das Antas Biological Reserve, Rio de Janeiro State, Brazil (REBIO Poço; $22^{\circ}$ $30^{\prime}-33^{\prime} \mathrm{S}, 42^{\circ} 15^{\prime}-19^{\prime} \mathrm{W}$ ) is situated in a fragment of Atlantic Coastal Forest and is covered with secondary forest in various stages of ecological succession (Dietz et al., 1997). All GLTs in ca. 20 reproductive groups in this reserve are dye-marked, tattooed, habituated to the presence of human observers and systematically monitored (Dietz and Baker, 1993; Baker and Dietz, 1996). The groups in this study area are native to this area and unmanipulated, except for semi-annual trapping to change radio collars and freshen dye marks. All births, death, immigrations, and emigrations in the study population are recorded.

During the past 20 years, as part of a successful reintroduction program, tamarins that were born in zoos around the world were returned to privately owned forest fragments in Rio de Janeiro State (Beck et al., 2002). Some of these farms are small isolated forest fragments; others have relatively large contiguous forests. The number of tamarins in the reintroduction area is estimated at 500. These GLTs are captured as necessary to dye mark individuals and replace radio collars.

\subsection{Golden lion tamarin sampling technique}

The capture methodology for the 199 GLTs in the present study was the same in both types of conservation units: family groups of GLTs were non-injuriously captured during the day using live-traps from June 2001 to June 2005. Captured individuals were transported to laboratories in the conservation units, anesthetized in the evening of the same day, and samples were collected during routine physical examinations. For each individual we recorded sex and body weight (BW). Fresh feces were collected from live traps and conserved in plastic vials containing $40 \mathrm{ml}$ of $4 \%$ formaldehyde until analysis. On the morning following capture, tamarins were returned to the capture location and released.

From the 199 individual GLTs sampled, 139 came from REBIO Poço das Antas, equivalent to 40-63\% of its tamarin population (due to variation in tamarin population size during the study period). Sixty individuals were sampled in nine Reintroduction farms, representing approximately $12 \%$ of its total tamarin 
population. About $41 \%$ of the individual tamarins sampled in REBIO Poço das Antas were examined multiple times (up to six times/individual; 256 examinations at all), while tamarins from the Reintroduction farms were sampled once.

\subsection{Age distribution of sampled tamarins}

Birth dates were known for 176 individuals. Mean ages of tamarins in populations from the two conservation units were not significantly different (one-way multi-factorial ANOVA, $p>0.05$ ). Overall mean age was 3.1 years and $95 \%$ of the individuals were below 9.4 years old. Lion tamarins were divided into three age classes: dependent tamarins (below the $25 \%$ quartile range: 338 days old), non-reproductive adult GLTs (between the 25 and $75 \%$ quartile ranges), and reproductive individuals (above the $75 \%$ quartile range: 1619 days old).

\subsection{Intestinal helminths: fecal analysis}

Feces were analyzed using a modified Ritchie's technique. Plastic vials containing feces were weighed and feces plus dilutent were suspended, filtered trough a gauze mesh, returned to the same plastic vial and reweighed. Feces weight was obtained from the difference between the two measurements. The filtrate was then centrifuged for $10 \mathrm{~min}$ at $2500 \mathrm{rpm}$, after which the supernatant was removed and the remaining pellet resuspended with $5 \mathrm{ml}$ of distillated water and $5 \mathrm{ml}$ of sulfuric ether. The content was centrifuged for $2 \mathrm{~min}$ at $1500 \mathrm{rpm}$, after which the supernatant was discarded and remaining pellet was re-suspended in $4 \%$ formaldehyde to a final volume of $1.0 \mathrm{ml}$. Eighty microliters of the final solution were dropped between slide and a $22 \mathrm{~mm} \times 22 \mathrm{~mm}$ glass coverslip, and all helminth eggs were counted under a $400 \times$ magnification objective. Fecal egg count for each helminth species (in eggs per gram of feces) was calculated for each sample according to the following formula: FEC $=(\mathrm{HEC} \times 100 / 8) /(\mathrm{FW})$, where FEC, fecal egg count; HEC, helminth egg count; $\mathrm{FW}$, feces weight in grams.

The negative binomial distribution (Fisher, 1941) is defined as $s^{2}=m+m^{2} / k$. The coefficient $k$ is thus an inverse measure of parasite aggregation across individuals in the host population (lower $k$ meaning that most parasites are concentrated in few individuals) (Gregory and Woolhouse, 1993; Shaw et al., 1998), and its best estimate is the following formula for corrected moment estimate of $k$ (Wilson et al., 2002): $k=\left(m^{2}-s^{2} / n\right) /$ $\left(s^{2}-m\right)$, where $m$ is the mean egg count (for each helminth species), $s^{2}$ the variance of each helminth species egg count and $n$ is the number of positive fecal samples for each helminth species.

Helminth eggs were identified in accordance with Sloss et al. (1999) and helminth classification follows Amin (1985) for acanthocephala, and Vicente et al. (1997) for nematodes. One acanthocephalan species, Oncicola sp., and five nematode species, Ancylostomatidae, Ascarididae, Oxyuridae, Spiruridae, and Trichostrongylidae comprised the helminth fauna identified in a previous assessment in golden lion tamarins (Monteiro et al., 2003).

We estimated fecal examination sensitivity by using the frequency of false negatives obtained from tamarins captured at least three times (38 individuals: 18 males, 20 females). In this set, we counted tamarins with a negative fecal exam between (after and before) two or more positive fecal exams for the same parasite species (i.e., we failed to find a parasite known to be present).

\subsection{Statistical analysis}

We estimated overall helminth prevalence, i.e., the proportion of tamarins infected with any helminth species, from the ratio of individuals with a positive fecal exam to total number of tamarins sampled. We used similar calculations to determine the prevalence of each helminth species. The observed prevalences of infections with multiple helminth species $(n=316$ samples) were compared to the expected frequencies for each helminth mixed-infection pair. Assuming that such infections occur by chance, expected frequencies were calculated by multiplying individual prevalences for all possible combinations of mixed infections $(n=199$ individuals). Golden lion tamarin sex, age and conservation unit of origin were tested as factors that might generate heterogeneity in helminth prevalence. We used the Fisher Exact Test to perform all prevalence comparisons. We assessed the relationship between sex, age class, conservation unit and helminth mean egg counts using ANOVA; feces weight was included as a covariate in these models. We used a significance level of $\alpha=0.05$ for all statistical tests.

\section{Results}

\subsection{Helminth prevalence in golden lion tamarins}

Presumed false negatives occurred in relation to Oncicola sp., Spiruridae and Trichostrongylidae in 11, 16 and $8 \%$ of the tamarin samples, respectively. Thus, we estimated that fecal examinations based on single 
Table 1

Prevalences of intestinal helminths of Leontopithecus rosalia, grouped by host sex and area of occurrence

\begin{tabular}{|c|c|c|c|c|c|c|c|c|c|}
\hline \multirow[t]{2}{*}{ Conservation unit } & \multirow[t]{2}{*}{ Sex } & \multirow[t]{2}{*}{$n$ Sampled } & \multirow[t]{2}{*}{ Overall prevalence $(\%)$} & \multicolumn{6}{|l|}{$(\%)$} \\
\hline & & & & Anc & Asc & Onc & Oxi & Spi & Tri \\
\hline \multirow[t]{3}{*}{ REBIO Poço das Antas } & Male & 76 & 63 & 13 & 03 & 29b & 05 & 32 & 32 \\
\hline & Female & 63 & 73 & 13 & 0 & $44 b$ & 05 & 35 & 41 \\
\hline & Total & 139 & $68 a$ & 13 & 01 & $36 c$ & 05 & 33e & $36 f$ \\
\hline \multirow[t]{3}{*}{ Reintroduction farms } & Male & 37 & 38 & 11 & 0 & 11 & 0 & 03 & 16 \\
\hline & Female & 23 & 48 & 04 & 0 & 22 & 0 & 04 & 26 \\
\hline & Total & 60 & $42 \mathbf{a}$ & 08 & 0 & $15 c$ & 0 & 03e & $20 f$ \\
\hline \multirow[t]{2}{*}{ Total } & Males & 113 & 55 & 12 & 02 & 23d & 04 & 22 & 27 \\
\hline & Females & 86 & 66 & 10 & 0 & 38d & 04 & 27 & 37 \\
\hline Total tamarins sampled & & 199 & 60 & 12 & 01 & 30 & 04 & 24 & 31 \\
\hline
\end{tabular}

Values are percentage of tamarins (in each row) that present at least one positive result in one or more captures ranging from June 2001 to 2005. For each helminth column, cells in bold highlight statistically different prevalences (tested with Fisher Exact Test) between factors (sex or conservation unit, or sex within conservation units), with different letters attributed to each pair of differences. Significance levels are as follows: (a) $p=0.001$; (b) $p=0.043$; (c) $p=0.002$; (d) $p=0.014$; (e) $p<0.001$; (f) $p=0.018$. Helminth egg codes: Anc, Ancilostomatidae sp.; Asc, Ascarididae sp.; Onc, Oncicola sp.; Oxi, Trypanoxyiuris minutus; Spi, Spiruridae; Tri, Trichostrongylidae.

samples had a mean sensitivity of $85 \%$ in the sampled populations, for all helminth species except Oxyuridae. There was no significant correlation between FEC and FW, indicating that the counts are unbiased by the amount of collected feces.

Helminth intestinal diversity in GLTs comprised six different species (Table 1). Three helminth species, Oncicola sp., Spiruridae and Trichostrongylidae had higher prevalences $(>30 \%)$ and were found in all conservation units. The Ancylostomatidae, Ascarididae and Oxyuridae species had relatively low prevalences $(<15 \%)$, were not found in all conservation units and were not considered in additional analyses. One adult of the Oxyuridae species was collected from a GLT and was identified as Tripanoxyuris minutus.

The proportion of tamarins positive for helminth species varied between sexes. Males had a significantly lower prevalence for Oncicola sp., and trends toward lower prevalences for overall and Trichostrongylidae infections. Higher overall prevalence in REBIO Poço das Antas was caused by high prevalences of Oncicola sp., Spiruridae and Trichostrongylidae helminths.

Risk of intestinal helminth infection starts early in the life of a GLT: we found all helminth species parasitizing at least one tamarin at 6 months of age or younger. There is an age-linked decrease in helminth prevalence that follows different patterns in male and female GLTs. Males presented peaks of helminth prevalence at ages 1 and 4 years old. After the first peak, prevalence fell for 2 years, rose suddenly at age four, and then decreased to minimum levels in ages thereafter. We also found two peaks of prevalence in females, but there was only a slight decrease in helminth prevalence between them (Fig. 1). At ages less than 5 years old, parasitized female tamarins showed higher peaks than males; helminth prevalences in both sexes were similar from this age on. The earlier and higher peaks of prevalence were related to Oncicola sp. infection; peaks of prevalence in Spiruridae and Trichostrongylidae occurred later and at lower levels.

\subsection{Helminth mixed infections}

The observed prevalence of mixed infection of Oncicola sp. $\times$ Trichostrongylidae in GLTs $(3.5 \%)$ was statistically lower than expected by chance alone $(9.3 \%$, Pearson Chi-square, $p<0.001, n=316$ ), and we found no sex bias. We found no other evidence of interactions such as competition or mutualism among helminths from GLTs; the prevalences calculated for each mixedinfection set of helminths did not differ from that predicted by chance.

\subsection{Helminth egg output in golden lion tamarins}

The mean helminth egg output (Table 2) did not vary significantly between conservation units (REBIO Poço das Antas versus Reintroduction farms) or sexes. The coefficient $k$ calculated for the three species indicates an aggregate pattern of distribution in the host population. Females had a significantly lower $k$ (i.e., higher aggregations) than did males relative to Trichostrongylidae helminths, and there was a similar trend in Spiruridae. The FEC of Oncicola sp., Spiruridae and 


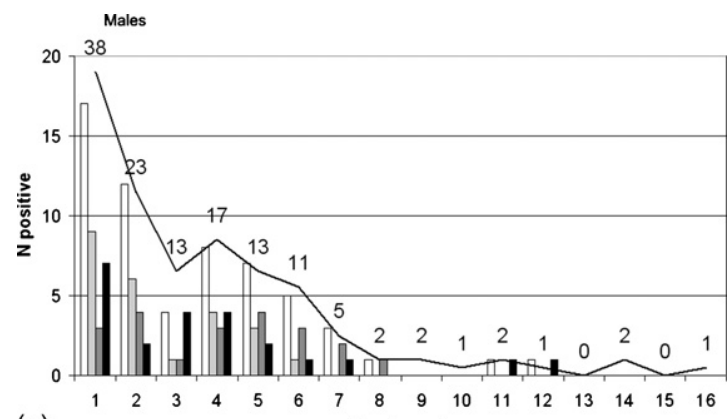

(a)

Age (years)

$\square$ Overall prevalence $\square$ Oncicola sp. $\square$ Spiruridae $\square$ Trichostrongilidae

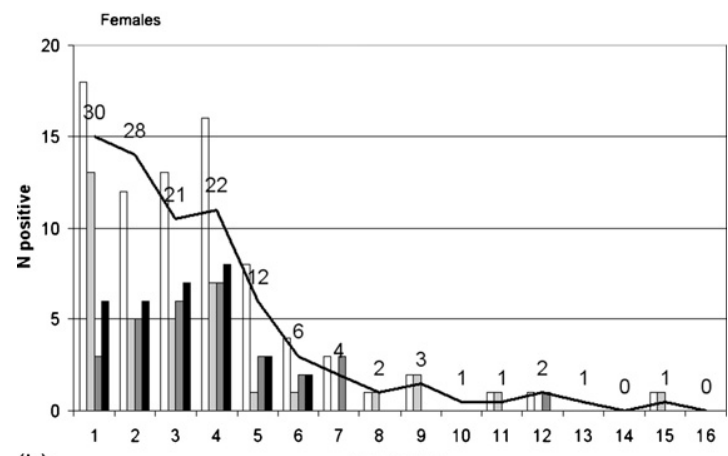

(b)

Age (years)

$\square$ Overall prevalence $\square$ Oncicola sp. $\square$ Spiruridae $\square$ Trichostrongilidae

Fig. 1. Age-distribution (in years) of helminth prevalence (counts) in male (panel a) and female (panel b) golden lion tamarins. Figures depict distribution of 139 positive fecal samples (out of 265 fecal samples from tamarins of known ages). Bars are frequency of overall prevalence (taking into account all helminth species), Oncicola sp., Spiruridae and Trichostrongylidae infection. The line refers to the number of samples in each age. Sample period: June 2001-2005.
Trichostrongylidae were statistically independent of age class (ANOVA; Fig. 2).

\subsection{Helminth infection and survival}

In order to evaluate if helminth infection can affect GLT survival, we used an ANOVA to test for a relationship between the mean ages of uninfected tamarins and those carrying Oncicola sp., Spiruridae, and Trichostrongylidae helminths. We included only the samples from animals beyond 2-years old, the age at which most positive samples were concentrated. We found that Trichostrongylidae infection was associated with lower mean age. The mean age (in days) of Trichostrongylidae-positive GLTs $(1440 \pm 803, n=34)$ was more than a year less than that of negative individuals $(1881 \pm 1041, n=112 ; F=5.158, p=0.025)$.

\section{Discussion}

The difference in diversity of helminth species between the conservation units seems to be an effect of sample size, as the helminth species absent in the Reintroduction farms are also those occurring at low prevalences in REBIO Poço das Antas. The 26\% higher overall helminth prevalence in REBIO Poço das Antas is attributable not only to its higher parasite diversity, but also due to greater prevalences of Oncicola sp., Spiruridae and Trichostrongylidae. This highlights a key aspect of host-parasite relationships: localities separated by a relatively short distance, $60 \mathrm{~km}$ in this study, can present sharp differences in helminth occurrence; Chapman et al. (2006) presented a similar

Table 2

Mean fecal egg counts (eggs/g) and $k$ estimate of aggregation of intestinal helminths found in golden lion tamarins, grouped by host sex and area of occurrence

\begin{tabular}{|c|c|c|c|c|c|c|c|}
\hline Conservation unit & Sex & Onc FEC & Onc $k$ & Spi FEC & Spi $k$ & Tri FEC & Tri $k$ \\
\hline \multirow[t]{3}{*}{ REBIO Poço das Antas } & Males & $29 \pm 49(20)$ & & $14 \pm 21(26)$ & & $45 \pm 79(24)$ & \\
\hline & Females & $25 \pm 45(23)$ & & $20 \pm 37(29)$ & & $46 \pm 130(30)$ & \\
\hline & Total & $27 \pm 46(43)$ & & $17 \pm 30(55)$ & & $45 \pm 109(54)$ & \\
\hline \multirow[t]{3}{*}{ Reintroduction Farms } & Males & $22 \pm 33(3)$ & & $2(1)$ & & $89 \pm 143(6)$ & \\
\hline & Females & $50 \pm 56(3)$ & & $2(1)$ & & $54 \pm 111(6)$ & \\
\hline & Total & $36 \pm 44(6)$ & & $2 \pm 0.2(2)$ & & $71 \pm 124$ (12) & \\
\hline \multirow[t]{3}{*}{ Total } & Males & $28 \pm 47(23)$ & 0.315 & $13 \pm 20(27)$ & 0.398 & $54 \pm 94(30)$ & $0.299^{\mathrm{a}}$ \\
\hline & Females & $28 \pm 46(26)$ & 0.336 & $19 \pm 36(30)$ & 0.249 & $47 \pm 126(36)$ & $0.112^{\mathrm{a}}$ \\
\hline & Total & $28 \pm 46(49)$ & 0.355 & $16 \pm 30(57)$ & 0.272 & $50 \pm 112(66)$ & 0.185 \\
\hline
\end{tabular}

Data are mean number of eggs per gram of feces plus or minus standard deviation, with the number of samples analyzed in parentheses. For $k$ calculations, see text. No mean was considered statistically different, through one-way nested ANOVA, $\alpha=0.05$. Helminth egg codes: Onc, Oncicola sp.; Spi, Spiruridae; Tri, Trichostrongilidae.

${ }^{a}$ Variance ratio test, $F=2.67,30 / 36$ d.f., $p<0.005$. 

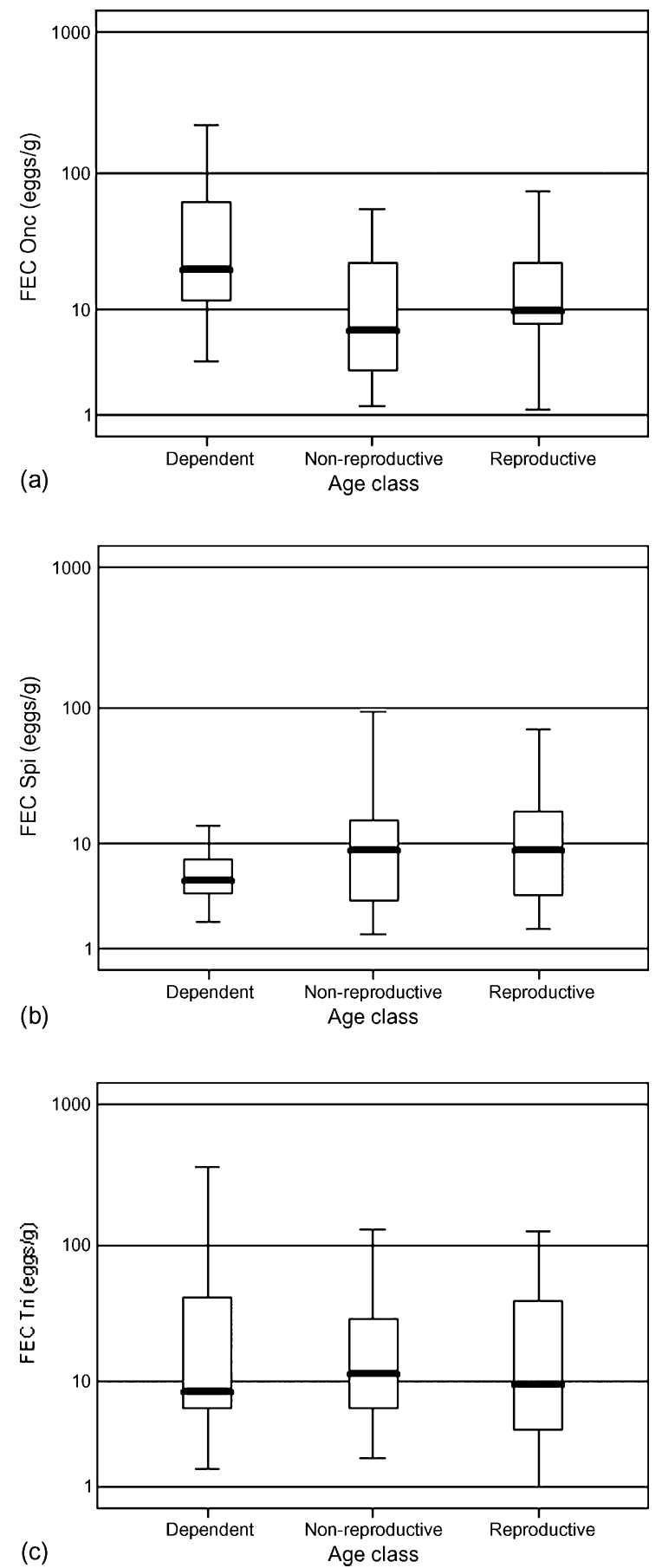

Fig. 2. Mean fecal egg count of three helminth species, Oncicola sp. (panel a), Spiruridae (panel b) and Trichostrongylidae (panel c) grouped by golden lion tamarin age classes. No mean was statistically different among age classes (ANOVA). The thick horizontal line is the median value, box represents percentiles $25-75 \%$, and error bars percentiles 5-95\%. finding in red and black-and-white colobus monkeys from Uganda.

Differences in helminth prevalence between conservation units may be explained by differences in rates of exposure to infective stages, rates of helminth elimination by the host and host death rate due to helminth infection (Gulland, 1995). GLTs have been present in REBIO Poço das Antas for at least 20,000 years (Rylands et al., 1996), co-evolving with their helminth parasites, and subject to selection for helminth resistance. In contrast, virtually all GLTs on the Reintroduction farms were descendents of zoo-born family groups that were helminth-free for several generations prior to release in wild areas; currently, nearly $95 \%$ of GLTs on Reintroduction farms are wildborn descendents of reintroduced animals (Beck et al., 2002). In these areas primate hosts have been locally extinct for about 30 years. Over time, the absence of an appropriate primate host may have lowered the availability of helminth infective stages in such farms, both in the intermediate invertebrate hosts (for Oncicola sp. and Spiruridae) and in the soil (for Trichostrongylidae). The difference in prevalence observed between Reintroduction farms and REBIO Poço das Antas may reflect a helminth recolonization process in the Reintroduction areas. The possibility that GLTs from these two conservation units have differential helminth elimination rates or helminth-linked death rates cannot be ruled out.

The data presented herein allow us to suggest that helminth prevalence in GLTs was affected by four factors: host age and sex, geographic area of occurrence, and helminth transmission strategy. The decrease in the number of GLTs sampled through increasing age is correlated with their helminth prevalence (Fig. 1), and with differences between sexes. We cannot ascertain if young GLTs died as a direct result of parasite infection (finding a dead GLT in the forest environment is difficult due to rapid decomposition or ingestion by scavengers). The low immigration rate in GLT groups (0.48 individual/group/ year; Baker and Dietz, 1996) does not support the hypothesis that emigration alone causes the observed bias in the age frequencies. In light of the higher parasite prevalences found in tamarins under 4 years old, it is likely that helminthic disease increases the mortality rate directly and/or in conjunction with other environmental factors (predation, low nutritional status, group size and composition).

The effects of sex and age on helminth prevalences (Fig. 1) are consistent with predictions that sex steroids affect immunocompetence (Hamilton and Zuk, 1982; 
Wilson et al., 2002; Klein, 2004; Bouman et al., 2005; Lamason et al., 2006). Two points in a male tamarin's life may be critical in this regard: from 1 to 2-years old (during sexual differentiation in males-as demonstrated in S. mystax; Huck et al., 2005), and after 4 years old, when becoming reproductive increases energetic requirements (Dietz et al., 1994). At these ages, increased energetic requirements and a lower immunologic status caused by the expression of sex steroids may cause an increase in host death rate. In female GLTs, prevalence of Spiruridae and Trichostrongylidae peaked at 4 years of age and decreased thereafter (Fig. 1). The metabolic demands elicited by helminth infection, added to the physiological demands of female emigration (most GLT daughters are expelled from their family groups at about 2 years old; Baker and Dietz, 1996) or pregnancy/lactation (when progesterone levels rise; French et al., 2003), may result in higher mortality in females after this age.

In captive callitrichids, Oncicola sp. attachment to the intestinal wall may cause severe pathogenic effects (gut perforation and/or inflammation, secondary bacterial invasion and peritonitis) or death (Toft, 1982). We expect that the same is possible in wild animals. However, acantocephalans absorb nutrients by diffusion through their cuticle, a relatively slow process (Crompton and Nickol, 1985), thus their use of tamarin metabolic resources is low. Low aggregation in the host population (without sex-associated differences) and low production of eggs without significant age class differences (but a tendency toward higher production in dependent tamarins, Fig. 2, panel a) are in accord with a mechanical rather than exploitative pathogenic effect in host GLTs. Host survival may be increased more by innate immunity than acquired immunity.

In contrast, the Spiruridae and Trichostrongylidae helminths probably exert negative selective pressure through resource exploitation (Toft, 1982). The extent of organic injury correlates with the number of parasites infecting the host, not only the presence or absence in the host (as is the case with Oncicola sp.). In Spiruridae and Trichostrongylidae infections, host survival may be increased more by acquired immunity than innate immunity. Our findings suggest that Spiruridae is less pathogenic then Trichostrongylidae in this host species; Spiruridae has double peaks of prevalence, low egg production, absence of differences in FEC among sex and age classes, and low aggregation (similar to Oncicola sp.).

Trichostrongylidae, a geohelminth, can be considered the most pathogenic of the helminths assessed in this study. Field studies suggest that Trichostrongylidae are capable of limiting the size of other wild animal populations. For example, Hudson et al. (2002) determined that Trichostrongylus tenuis was the parasite responsible for the cyclic fluctuations in abundance of red grouse (Lagopus lagopus) in Scotland. The population densities of red grouse treated with antihelminthic drugs remained stable, while untreated populations fluctuated. Newey et al. (2005) points to the possibility that Trichostrongylus retortaeformis may be partially responsible for the 7-10 year fluctuations in population size of mountain hares (Lepus timidus) in Scotland. GLTs occasionally descend to the ground to travel or forage, thus tamarins living in more fragmented forest environments may spend more time on the ground and in greater contact with Trichostrongylidae eggs. The presence of Trichostrongylidae infection is associated with lower mean ages of infected individuals (see Section 3.4). Removal of young tamarins from the analysis partially corrects for the higher exposure at these ages, but we cannot assume that reduced survivorship of host population is a characteristic of Trichostrongylidae infection alone. We suggest that it may be exacerbated by low environmental resources in those areas.

The sex difference in Trichostrongylidae aggregation indicates that females are better able to afford the higher energetic expenditure represented by the Trichostrongylidae egg output, i.e., males may suffer higher mortality than females under similar levels of Trichostrongylidae egg production. The lower energetic cost caused by Oncicola sp. and Spiruridae egg production allows a lower and similar aggregation level in both sexes in tamarins parasitized by those helminth species. The tendency for female tamarins to have higher Spiruridae aggregation re-enforces this hypothesis, as pathogenicity due to this helminth is also linked to the extent of host colonization.

Differences in the coefficient $k$ among helminths may also reflect a difference in their dispersion strategies: Oncicola sp. and Spiruridae, species that rely on invertebrate vectors to disperse, had the highest coefficients, indicating a more even distribution of parasites across individuals in the population. The loss of infective stages in this mode of dispersion is minimized by ingestion of feces by intermediate vectors, positively favoring selection for helminth genetic lineages where egg production is not higher, but of greater duration. The Trichostrongylidae species had the lowest value of $k$, indicating a higher clumping of parasites in few host individuals, a pattern that is consistent with their direct life cycle. In this manner of dispersion, environmental loss of infective stages is 
relatively high, so helminth genetic lineages that maximize production of infective stages (i.e., eggs) inside the host would be positively selected for under natural conditions. As the production of helminth infective stages is accomplished at the expense of host resources, pathogenicity of such species may result in part from their need to maximize transmission. Note in Table 2 that Trichostrongylidae egg output is twice that of the two other species, the smaller size of their eggs notwithstanding (Monteiro et al., 2003).

Three different reasons may explain the statistically lower frequency of association of the Oncicola sp. $\times$ Trichostrongylidae pair in GLTs. The simultaneous occurrence of these helminths could increase host death rate due to higher pathogenicity, thus lowering the observed frequency of association. Second, competition between these helminths could lower their simultaneous occurrence. Third, environmental factors may influence the risk of exposure to these helminth species in different ways. Low availability of invertebrates in fragmented ("patchy") areas could reduce contact with Oncicola sp. infective stages while promoting a higher contact with Trichostrongylidae eggs. In areas with more contiguous forest, the density of invertebrate vectors may be higher thus favoring transmission of Oncicola sp. and diminishing the exposure of GLTs to the eggs of Trichostrongylidae located in the soil.

Our data suggest that infection by Trichostrongylidae species, as well as Oncicola sp. and Spiruridae species, may act as a selective force that biases the host abundance curve over time (Tompkins et al., 2002). In cases of low host abundance (small and isolated populations), tamarin population birth rates could reach lower levels than death rates imposed by Oncicola sp. species, in which infection is facilitated through continuous ingestion of intermediary invertebrate hosts, a major part of tamarins' daily diet. In contrast, in areas with high abundance of GLTs (as in REBIO Poço das Antas) the accumulation of Spiruridae in the host and invertebrate vector populations might lead to an environmental over-exposure to this helminth, increasing their and Trichostrongylidae helminths' pathogenic effects. This synergism might produce fluctuations in GLT population size capable of causing extinction or significant loss of genetic diversity due to reduction in population size.

\section{Acknowledgements}

We thank the director and staff of IBAMA in REBIO Poço das Antas for logistic support, the owners of Reintroduction farms for access to their properties, and the director and staff of the Associação Mico Leão Dourado for logistic and technical support.

This work was supported by grants from the Brazilian Conselho Nacional de Pesquisa e Desenvolvimento - CNPq, Edital Universal no. 472777/2003, and the US National Science Foundation.

This work was conducted under authorization from IBAMA, $\mathrm{CNPq}$ and the Animal Care and Use Committee of FIOCRUZ.

\section{References}

Amin, O.M., 1985. Classification. In: Crompton, D.W.T., Nickol, B.B. (Eds.), Biology of the Acanthocephala. University Press, Cambridge, pp. 22-71.

Anderson, R.M., May, R.M., 1985. Helminth infections of humans: mathematical models, population dynamics, and control. Adv. Parasitol. 24, 1-101.

Baker, A.J., Dietz, J.M., 1996. Immigration in wild groups of golden lion tamarins (Leontopithecus rosalia). Am. J. Primatol. 38, 47-56.

Beck, B., Castro, M.I., Stoinski, T.S., Ballou, J., 2002. The effects of prerelease environments and postrelease management on survivorship in reintroduced golden lion tamarins. In: Kleiman, D.G., Rylands, A.B. (Eds.), Lion Tamarins: Biology and Conservation. Smithsonian Institution Press, Washington, DC, pp. 283-300.

Bouman, A., Heineman, M.J., Faas, M.M., 2005. Sex hormones and the immune response in humans. Hum. Reprod. Update 11 (4), 411-423.

Chapman, C.A., Speirs, M.L., Gillespie, T.R., Holland, T., Austad, K.M., 2006. Life on the edge: gastrointestinal parasites from the forest edge and interior primate groups. Am. J. Primatol. 68, 397409.

Crompton, D.W.T., Nickol, B.B., 1985. Biology of the Acanthocephala. University Press, Cambridge, 519 pp.

Dietz, J.M., Baker, A.J., 1993. Polygyny and female reproductive success in golden lion tamarins, Leontopithecus rosalia. Anim. Behav. 46, 1067-1078.

Dietz, J.M., Baker, A.J., Miglioretti, D., 1994. Seasonal variation in reproduction, juvenile growth, and adult body mass in golden lion tamarins (Leontopithecus rosalia). Am. J. Primatol. 34, 115-132.

Dietz, J.M., Peres, C.A., Pinder, L., 1997. Foraging ecology and use of space in wild golden lion tamarins (Leontopithecus rosalia). Am. J. Primatol. 41, 289-305.

Fisher, R.A., 1941. The negative binomial distribution. Ann. Eugen. 11, 182-187.

French, J.A., Bales, K.L., Baker, A.J., Dietz, J.M., 2003. Endocrine monitoring of wild dominant and subordinate female Leontopithecus rosalia. Int. J. Primatol. 24 (6), 1281-1300.

Gregory, R.D., Woolhouse, M.E., 1993. Quantification of parasite aggregation: a simulation study. Acta Trop. 54 (2), 131-139.

Gulland, F.M.D., 1995. The impact of infectious diseases on wild animal populations: a review. In: Grenfell, B.T., Dobson, A.P. (Eds.), Ecology of Infectious Diseases in Natural Populations. Cambridge University Press, Cambridge, pp. 20-51.

Hamilton, W.D., Zuk, M., 1982. Heritable true fitness and bright birds: a role for parasites. Science 218, 384-387.

Huck, M., Löttker, P., Heymann, E.W., Heistermann, M., 2005. Characterization and social correlates of fecal testosterone and cortisol excretion in wild male Saguinus mystax. Int. J. Primatol. 26 (1), 159-179. 
Hudson, P.J., Dobson, A.P., Newborn, D., 2002. Prevention of population cycles by parasite removal. Science $282,2256-2258$.

IUCN, 2004. 2004 IUCN Red List of Threatened Species. $<$ www.redlist.org $>$. Downloaded on 20 May 2005.

Klein, S.L., 2004. Hormonal and immunological mechanisms mediating sex differences in parasite infection. Parasite Immunol. 26, 247-264.

Lamason, R., Zhao, P., Rawat, R., Davis, A., Hall, J.C., Chae, J.J., Agarwal, R., Cohen, P., Rosen, A., Hoffman, E.P., Nagaraju, K., 2006. Sexual dimorphism in immune response genes as a function of puberty. BMC Immunol. 7 (2) [Epub ahead of print].

Monteiro, R.V., Jansen, A.M., Pinto, R.M., 2003. Coprological helminth screening in Brazilian free ranging golden lion tamarins, Leontopithecus rosalia (L., 1766) (Primates, Callitrichidae). Braz. J. Biol. 63 (4), 727-729.

Myers, N., Mittermeier, R.A., Mittermeier, C.G., da Fonseca, G.A.B., Kent, J., 2000. Biodiversity hotspots for conservation priorities. Nature 403, 853-858.

Newey, S., Shaw, D.J., Kirby, A., Montieth, P., Hudson, P.J., Thirgood, S.J., 2005. Prevalence, intensity and aggregation of intestinal parasites in mountain hares and their potential impact on population dynamics. Int. J. Parasitol. 35, 367-373.

Poulin, R., 2001. Interactions between species and the structure of helminth communities. Parasitology 122, S3-S11.

Rylands, A.B., da Fonseca, G.A.B., Leite, Y.L.R., Mittermeier, R.A., 1996. Primates of the Atlantic forest. In: Norconk, M.A., Ro- senberger, A.L., Garber, P.A. (Eds.), Adaptive Radiations of Neotropical Primates. Plenum Press, New York, pp. 21-51.

Shaw, D.J., Grenfell, B.T., Dobson, A.P., 1998. Patterns of macroparasite aggregation in wildlife host populations. Parasitology 117, 597-610.

Sloss, M.W., Zajac, A.M., Kemp, R.L., 1999. Parasitologia Clínica Veterinária. Manole, São Paulo, 198 pp.

Toft 2nd, J.D., 1982. The pathoparasitology of the alimentary tract and pancreas of nonhuman primates: a review. Vet. Pathol. Suppl. 7, 44-92.

Tompkins, D.M., Dobson, A.P., Arneberg, P., Begon, M.E., Cattadori, I.M., Greenman, J.V., Heesterbeek, J.A.P., Hudson, P.J., Newborn, D., Pugliese, A., Rizzoli, A.P., Rosà, R., Rosso, F., Wilson, K., 2002. Parasites and host population dynamics. In: Hudson, P.J., Rizzoli, A., Grenfell, B.T., Heesterbeek, H., Dobson, A.P. (Eds.), The Ecology of Wildlife Diseases. Oxford, New York, pp. 45-62.

Vicente, J.J., Rodrigues, H.O., Gomes, D.C., Pinto, R.M., Faria, Z., 1997. Nematóides do Brasil, parte V: nematóides de mamíferos. Rev. Bras. Zool. 14 (Suppl. 1), 1-452.

Wilson, K., Bjørnstad, O.N., Dobson, A.P., Merler, S., Poglayen, G., Randolph, S.E., Read, A.F., Skorping, A., 2002. Heterogeneities in macroparasite infections: patterns and processes. In: Hudson, P.J., Rizzoli, A., Grenfell, B.T., Heesterbeek, H., Dobson, A.P. (Eds.), The Ecology of Wildlife Diseases. Oxford, New York, pp. 6-44.

Woolhouse, M.E.J., 1998. Patterns in parasite epidemiology: the peak shift. Parasitol. Today 14 (10), 428-434. 